\title{
Adsorption-Driven Photocatalytic Activity of Mesoporous Titanium Dioxide
}

Yasuhiro Shiraishi, "Naoya Saito, and Takayuki Hirai

Research Center for Solar Energy Chemistry, and Division of Chemical Engineering,

Graduate School of Engineering Science, Osaka University, Toyonaka 560-8531, Japan

\section{Supporting Information}

\section{Materials and Methods}

\section{Catalyst Preparation}

$m \mathrm{TiO}_{2}(x)[x=65,61,37,0]$. These materials were synthesized according to literature procedure, ${ }^{\mathrm{S} 1}$ as follows: $1.0 \mathrm{~g}$ of Pluronic 123 triblock copolymer $\left(\mathrm{P} 123 ; \mathrm{EO}_{20} \mathrm{PO}_{70} \mathrm{EO}_{20} ; M=\right.$ 5800) was dissolved in dry ethanol (20 g). Titanium tetrachloride ( $\left.\mathrm{TiCl}_{4} ; 0.6 \mathrm{~g}\right)$ and titanium tetraisopropoxide $\left(\mathrm{Ti}\left(\mathrm{OPr}^{i}\right)_{4} ; 2.5 \mathrm{~g}\right)$ were added to the solution, and the mixture was stirred for $2 \mathrm{~h}$ at room temperature. The resulting mixture was transferred to a petri dish and dried at $313 \mathrm{~K}$ for $24 \mathrm{~h}$. For $m \mathrm{TiO}_{2}(0)$, the remaining surfactant was removed by the Soxhlet extraction using acetonitrile for $12 \mathrm{~h}$. For other materials, the surfactant was removed by calcination at $623 \mathrm{~K}$, for 2 $\mathrm{h}$ under air flow $\left[m \mathrm{TiO}_{2}(37)\right]$, for $4 \mathrm{~h}$ under $\mathrm{O}_{2}$ flow $\left[m \mathrm{TiO}_{2}(61)\right]$, and for $6 \mathrm{~h}$ under air flow $\left[m \mathrm{TiO}_{2}(65)\right]$, respectively, affording white powders.

$\operatorname{agg} \mathrm{TiO}_{2}(\mathbf{3 6})$. This material was synthesized according to literature procedure, ${ }^{\mathrm{S} 2}$ as follows: aqueous $\mathrm{H}_{2} \mathrm{O}_{2}$ solution (30 wt \%; $\left.13.6 \mathrm{~g}\right)$ was added slowly to ethanol $(10 \mathrm{~mL})$ containing $\operatorname{Ti}\left(\mathrm{OPr}^{i}\right)_{4}(1 \mathrm{M})$ with stirring. An aqueous solution of D-mannitol $(20 \mathrm{mM}, 100 \mathrm{~mL})$ was then added to the mixture. The solution was heated at $368 \mathrm{~K}$ for $24 \mathrm{~h}$ with stirring in a glass beaker capped with a rubber septum to suppress the evaporation of solvent, where several small holes were made on the septum to release the oxygen formed by decomposition of $\mathrm{H}_{2} \mathrm{O}_{2}$. The obtained gel was dispersed into water $(500 \mathrm{~mL})$, stirred for $1 \mathrm{~h}$ at $348 \mathrm{~K}$, and recovered by filtration. This procedure was repeated three times. The resulting gel was then dried at $348 \mathrm{~K}$ for $12 \mathrm{~h}$, affording a white powder. 
$\boldsymbol{m} \mathrm{TiO}_{2}(\mathbf{5 7})$. This was obtained by a calcination of $\operatorname{agg} \mathrm{TiO}_{2}(36)$ at $623 \mathrm{~K}$ for $0.5 \mathrm{~h}$ under air flow as a white powder, according to literature procedure. ${ }^{\mathrm{S} 2}$

$\boldsymbol{n} \mathbf{T i O}_{2}(\mathbf{0})$. This material (amorphous form) was supplied from Wako Pure Chemical Industries, Ltd., which was used for photocatalytic reactions after drying at $423 \mathrm{~K}$ for $12 \mathrm{~h}$ under vacuum.

$n \mathbf{T i O}_{2}(\mathbf{5 8})$. This was obtained by a calcination of $n \mathrm{TiO}_{2}(0)$ at $623 \mathrm{~K}$ for $6 \mathrm{~h}$ under air flow, as a white powder.

$\boldsymbol{n} \mathbf{T i O}_{2}(\mathbf{1 0 0})$. This was obtained by a calcination of $n \mathrm{TiO}_{2}(0)$ at $623 \mathrm{~K}$ for $18 \mathrm{~h}$ under air flow, as a white powder.

\section{Photocatalytic Reaction}

Each catalyst $(10 \mathrm{mg})$ was suspended in a buffered aqueous solution $(\mathrm{pH} 7 ; 10 \mathrm{~mL}$; consisting of $0.05 \mathrm{M} \mathrm{KH}_{2} \mathrm{PO}_{4}$ and $\left.0.03 \mathrm{M} \mathrm{NaOH}\right)$ containing an individual substrate $(20 \mu \mathrm{mol})$ within a Pyrex glass tube (capacity: $20 \mathrm{~mL}$ ), and each tube sealed using a rubber septum cap. The catalyst was dispersed well by ultrasonication for $5 \mathrm{~min}$ and nitrogen was then bubbled through the solution for $10 \mathrm{~min}$. The sample was photoirradiated with stirring by a high-pressure Hg lamp (300 W, Eikohsha Co. Ltd., Osaka), filtered through an uranil glass (Eikohsha Co. Ltd., Osaka) to give light wavelengths of $\lambda>320 \mathrm{~nm}$. It was confirmed that all of the substrates used scarcely absorb the light at this range and almost no reaction of substrates proceeds in the absence of catalyst. The temperature of the solution during photoirradiation was $313 \mathrm{~K}$. After photoirradiation, the catalyst was recovered by centrifugation and washed successively with acetonitrile $(10 \mathrm{~mL})$, acetone $(10$ $\mathrm{mL})$, and water $(10 \mathrm{~mL})$ under ultrasonication. The combined solution was then analyzed by a reverse-phase HPLC (Shimadzu LC-6A) equipped with UV-vis spectrometric detector (Shimadzu SPD-6A; $\left.\lambda_{\text {abs }}=254 \mathrm{~nm}\right)$.

\section{Adsorption Experiment}

Each catalyst (10 mg) was suspended in a buffered aqueous solution ( $\mathrm{pH} 7 ; 10 \mathrm{~mL})$ containing 
an individual substrate $(20 \mu \mathrm{mol})$ within a Pyrex glass tube (capacity: $20 \mathrm{~mL}$ ), and each tube sealed using a rubber septum cap. The catalyst was dispersed well by ultrasonication for 5 min. Nitrogen was bubbled through the solution for $10 \mathrm{~min}$. The mixture was stirred for $0.5 \mathrm{~h}$ at $313 \mathrm{~K}$. The resulting solution was recovered by filtration and analyzed by HPLC.

\section{ESR Measurement}

ESR spectra were recorded at the X-band using a Bruker EMX-10/12 spectrometer with a 100 $\mathrm{kHz}$ magnetic field modulation at a microwave power level of $1.0 \mathrm{~mW}$, where microwave power saturation of the signals does not occur. The magnetic field was calibrated using a 1,1'-diphenyl-2-picrylhydrazyl (DPPH) as standard. Each $\mathrm{TiO}_{2}$ catalyst $(5 \mathrm{mg})$ was placed in a flat ESR cell $[10 \mathrm{~mm} \times 20 \mathrm{~mm} \times 3 \mathrm{~mm}$ (path length) $]$ and treated with 100 Torr oxygen $(1$ Torr $=$ 133.3 $\mathrm{Pa}$ ) at $473 \mathrm{~K}$ for $12 \mathrm{~h}$. The tube was then cooled to room temperature under vacuum and was filled with dry $\mathrm{N}_{2}$. $\alpha$-Phenyl- $N$-tert-butylnitrone (PBN), a spin trapping agent, was dissolved in a buffered aqueous solution ( $\mathrm{pH} 7 ; 5 \mathrm{mM})$ and the solution $(5 \mathrm{~mL})$ was introduced to the cell under dry $\mathrm{N}_{2}$. The cell was placed on an ESR sample cavity and was photoirradiated using a $500 \mathrm{~W}$ Xe lamp (USHIO Inc.) through CS 0-52 filter (Corning) to give light wavelengths of $\lambda>350 \mathrm{~nm}$ at room temperature. After photoirradiation for $0.5 \mathrm{~h}$, the irradiation was turned off, and the measurement was started immediately. 


\section{Discussion}

Lawless et al. ${ }^{\mathrm{S} 3}$ have reported that the reaction of $\cdot \mathrm{OH}$ with the surface of $\mathrm{TiO}_{2}$ particles $(133 \AA$ diameter) proceeds in a near-diffusion-controlled rate $\left(k_{1}=6.0 \times 10^{11} \mathrm{M}^{-1} \mathrm{~s}^{-1}\right)$ by a pulse radiolysis technique. The rate equation of the $\cdot \mathrm{OH}$ disappearance in a $\mathrm{TiO}_{2}$-suspended aqueous solution is expressed, as follows:

$$
\begin{gathered}
-\frac{d[\cdot \mathrm{OH}]}{d t}=k_{1}\left[\mathrm{TiO}_{2}\right]_{\mathrm{p}}[\cdot \mathrm{OH}] \\
{\left[\mathrm{TiO}_{2}\right]_{\mathrm{p}}=\frac{w}{\frac{4}{3} \pi R^{3} N d}=\frac{3 w}{4 \pi R^{3} N d}}
\end{gathered}
$$

, where $\left[\mathrm{TiO}_{2}\right]_{\mathrm{p}}$ is a number of $133 \AA \mathrm{TiO}_{2}$ particles in solution $(\mathrm{M}) ; w$ is the quantity of $\mathrm{TiO}_{2}$ particles in solution $\left(\mathrm{g} \mathrm{L}^{-1}\right) ; N$ is Avogadro's number; $d$ is the density of $\mathrm{TiO}_{2}\left(=3.84 \times 10^{6} \mathrm{~g} \mathrm{~m}^{-3}\right)$; and $R$ is the radius of the particle $(=66.5 \AA)$.

The reaction of $\mathrm{OH}$ with $\mathrm{TiO}_{2}$ proceeds on "surface" of the $\mathrm{TiO}_{2}$ particles, such that the reaction rate depends on the "surface area" of the particles. The eq. 1 can therefore be transformed into eq. 3 , which is based on the surface area of the $\mathrm{TiO}_{2}$ particles per unit volume of solution, $\left[\mathrm{TiO}_{2}\right]_{\mathrm{s}}$, as follows:

$$
\begin{aligned}
& -\frac{d[\cdot \mathrm{OH}]}{d t}=k_{1}^{\prime}\left[\mathrm{TiO}_{2}\right]_{\mathrm{S}}[\cdot \mathrm{OH}] \\
& {\left[\mathrm{TiO}_{2}\right]_{\mathrm{S}}=\frac{4 \pi R^{2}}{\frac{4}{3} \pi R^{3} d} \times w=\frac{3 w}{R d} \quad\left(\mathrm{~m}^{2} \mathrm{~L}^{-1}\right)}
\end{aligned}
$$

, where

$$
k_{1}\left[\mathrm{TiO}_{2}\right]_{\mathrm{p}}=k_{1}^{\prime}\left[\mathrm{TiO}_{2}\right]_{\mathrm{s}}
$$

Hence, the rate constant for the $\cdot \mathrm{OH}$ disappearance, $k_{1}$ ', can be calculated, as follows:

$$
k_{1}^{\prime}=\frac{k_{1}\left[\mathrm{TiO}_{2}\right]_{\mathrm{p}}}{\left[\mathrm{TiO}_{2}\right]_{\mathrm{s}}}=\frac{k_{1}}{4 \pi R^{2} N}=1.8 \times 10^{3} \quad\left(\mathrm{~L} \mathrm{~m}^{-2} \mathrm{~s}^{-1}\right)
$$

In the presently considered $m \mathrm{TiO}_{2}$ system, $\cdot \mathrm{OH}$ is formed mainly within the mesopores. We therefore assume that $\cdot \mathrm{OH}$ is formed inside a cylindrical $\mathrm{TiO}_{2}$ wall. The surface area of the $\mathrm{TiO}_{2}$ 
wall per unit pore volume of $m \mathrm{TiO}_{2}$ can be expressed as eq. 7 , based on the pore volume, $V_{\mathrm{p}}$, and internal surface area, $A_{\text {int }}$, of $m \mathrm{TiO}_{2}$. In that, $A_{\text {int }}$ is obtained by subtracting the external surface area $\left(A_{\text {ext }}\right)$ from the BET surface area $\left(A_{\mathrm{BET}}\right)$, as shown in Table 1.

$$
\left[\mathrm{TiO}_{2}\right]_{\mathrm{s}}=\frac{A_{\mathrm{int}}}{V_{\mathrm{p}}}=\frac{A_{\mathrm{BET}}-A_{\mathrm{ext}}}{V_{\mathrm{p}}}
$$

$\left[\mathrm{TiO}_{2}\right]_{\mathrm{s}}$ is significantly larger than $[\cdot \mathrm{OH}]$, such that $k_{1}{ }^{\prime}\left[\mathrm{TiO}_{2}\right]_{\mathrm{s}}$ in eq. 3 is regarded to be almost constant during photoreaction. The eq. 3 is therefore regarded as a pseudo-first order reaction relying on $[\cdot \mathrm{OH}]$. The lifetime of $\cdot \mathrm{OH}$ on $m \mathrm{TiO}_{2}$, $\tau$, can therefore be expressed as follows:

$$
\tau=\frac{1}{k_{1}^{\prime}\left[\mathrm{TiO}_{2}\right]_{\mathrm{S}}}
$$

Thus, the diffusion distance of $\cdot \mathrm{OH}$ within its lifetime, $x$, is expressed as follows ${ }^{\mathrm{S}}$ :

$$
x=\sqrt{D \tau}
$$

, where $D$ is the diffusion coefficient of $\cdot \mathrm{OH}$ in water $\left(=2.6 \times 10^{-9} \mathrm{~m}^{2} \mathrm{~s}^{-1}\right) .{ }^{\mathrm{S} 5}$ The lifetime of $\cdot \mathrm{OH}$ and its diffusion distance within the lifetime on $m \mathrm{TiO}_{2}$, calculated by the above equations, are summarized in Table S4. The results reveal that the lifetime of $\cdot \mathrm{OH}$ on $m \mathrm{TiO}_{2}$ is $0.6-2.3 \mathrm{~ns}$ and the diffusion distance within the lifetime is $1.3-2.4 \mathrm{~nm}$. As shown in Table 1, the value of the diffusion distance is smaller than the pore diameter of $m \mathrm{TiO}_{2}$. The results suggest that most of $\cdot \mathrm{OH}$, formed within the mesopores on $m \mathrm{TiO}_{2}$, scarcely diffuse outside of the pores and also that the reaction of $\cdot \mathrm{OH}$ with substrates occurs within the pore. 


\section{Tables}

Table S1. Properties of substrates.

\begin{tabular}{|c|c|c|c|c|c|c|c|c|c|c|c|c|c|c|}
\hline \multirow[b]{2}{*}{ No. } & \multirow[b]{2}{*}{ substrate } & \multirow[b]{2}{*}{$E_{1 / 2}^{o x}(\mathrm{~V})^{a}$} & \multirow[b]{2}{*}{$\begin{array}{c}\text { HOMO } \\
\text { level }(\mathrm{eV})^{b}\end{array}$} & \multirow[b]{2}{*}{$\log P^{c}$} & \multicolumn{2}{|c|}{$m \mathrm{TiO}_{2}(65)$} & \multicolumn{2}{|c|}{$m \mathrm{TiO}_{2}(61)$} & \multicolumn{2}{|c|}{$m \mathrm{TiO}_{2}(57)$} & \multicolumn{2}{|c|}{$m \mathrm{TiO}_{2}(37)$} & \multicolumn{2}{|c|}{$m \mathrm{TiO}_{2}(0)$} \\
\hline & & & & & $D^{d}$ & $\begin{array}{l}\text { conv } \\
(\%)^{e}\end{array}$ & $D^{d}$ & $\begin{array}{l}\text { conv } \\
(\%)^{e}\end{array}$ & $D^{d}$ & $\begin{array}{l}\text { conv } \\
(\%)^{e}\end{array}$ & $D^{d}$ & $\begin{array}{l}\text { conv } \\
(\%)^{e}\end{array}$ & $D^{d}$ & $\begin{array}{l}\text { conv } \\
(\%)^{e}\end{array}$ \\
\hline 1 & phenol & 1.60 & 0.292 & 1.64 & 0 & 3.3 & 0 & 5.2 & 0 & 2.2 & 0 & 1.0 & 0 & 1.3 \\
\hline 2 & 2,4,6-trichlorophenol & 1.84 & -0.441 & 3.87 & 0 & 18.7 & 0.04 & 16.9 & 0.02 & 2.8 & 0.04 & 10.9 & 0.02 & 3.2 \\
\hline 3 & chlorohydroquinone & 1.21 & -0.087 & 1.81 & 0 & 17.8 & 0 & 15.5 & 0.03 & 18.8 & 0.02 & 13.8 & 0.03 & 4.1 \\
\hline 4 & 2,4-dichlorophenol & 1.79 & -0.183 & 3.26 & 0 & 17.9 & 0.01 & 14.8 & 0 & 2.0 & 0 & 5.6 & 0.04 & 2.5 \\
\hline 5 & 3-chlorophenol & 1.76 & -0.100 & 2.5 & 0 & 16.7 & 0 & 12.6 & 0 & 3.2 & 0 & 7.4 & 0.51 & 8.1 \\
\hline 6 & 4-chlorophenol & 1.65 & 0.049 & 2.43 & 0.02 & 16.0 & 0 & 15.5 & 0 & 5.1 & 0 & 4.6 & 0.05 & 6.1 \\
\hline 7 & 2-chlorophenol & 1.71 & 0.029 & 2.16 & 0.03 & 13.2 & 0 & 14.3 & 0.11 & 12.1 & 0.11 & 17.4 & 0.35 & 4.7 \\
\hline 8 & benzyl alcohol & 1.38 & 0.138 & 1.02 & 0.03 & 14.6 & 0 & 13.5 & 0.02 & 11.1 & 0.03 & 11.5 & 0.02 & 8.4 \\
\hline 9 & $\begin{array}{l}\text { 2,4-dichlorophenoxyacetic } \\
\text { acid }\end{array}$ & 0.88 & -0.636 & 2.69 & 0.06 & 20.5 & 0.09 & 33.8 & 0.13 & 11.1 & 0.07 & 16.0 & 0.10 & 6.2 \\
\hline 10 & $p$-cresol & 1.49 & 0.330 & 2.13 & 0.07 & 16.6 & 0.02 & 18.2 & 0 & 13.2 & 0 & 12.7 & 0.05 & 5.1 \\
\hline 11 & phenoxyacetic acid & 0.71 & -0.141 & 1.31 & 0.08 & 21.6 & 0.11 & 33.1 & 0.21 & 18.1 & 0.10 & 16.5 & 0.16 & 5.8 \\
\hline 12 & 1,2,4-trihydroxybenzene & 0.97 & 0.137 & 0.87 & 0.09 & 20.8 & 0.18 & 70.2 & 0.22 & 23.5 & 0.26 & 19.3 & 0.10 & 7.3 \\
\hline 13 & 1,3,5-trihydroxybenzene & 1.36 & 0.256 & 0.87 & 0.16 & 29.6 & 0.12 & 40.2 & 0.01 & 6.1 & 0.07 & 10.7 & 0.07 & 7.1 \\
\hline 14 & 4-chlorophenoxyacetic acid & 0.75 & -0.433 & 1.73 & 0.19 & 39.2 & 0.12 & 25.8 & 0.19 & 21.4 & 0.16 & 18.1 & 0.16 & 8.2 \\
\hline 15 & $\begin{array}{l}\text { 2,6-bis(hydroxymethyl) } \\
\text { - } p \text {-cresol }\end{array}$ & 1.51 & 0.117 & 0.98 & 0.24 & 40.1 & 0.15 & 50.8 & 0.31 & 40.2 & 0.40 & 27.9 & 0.50 & 8.2 \\
\hline
\end{tabular}

${ }^{a}$ vs. $\mathrm{Ag} / \mathrm{AgCl}$ (in acetonitrile with $\left.\mathrm{TBABF}_{4}{ }^{-}\right) .{ }^{b}$ calculated by PM3 method within WinMOPAC ver.3.0 (Fujitsu Inc.). ${ }^{c}$ calculated by ChemDraw Ultra ver.7.0.1 (CambridgeSoft Corp.). ${ }^{d}$ time, $0.5 \mathrm{~h}$; temperature, $313 \mathrm{~K}$ (the same data as those in Figure $1 \mathrm{~B}$ ). ${ }^{e}$ photoirradiation time, $0.5 \mathrm{~h}$ (the same data as those in Figure 1B). 
Table S2. Examples of photocatalytic hydroxylation of benzene to phenol.

\begin{tabular}{|c|c|c|c|c|c|c|c|c|}
\hline entry & oxidant & photocatalyst & reaction conditions & reaction mixture composition & $\begin{array}{l}\text { conv } \\
(\%)\end{array}$ & $\begin{array}{l}\text { yield } \\
(\%)\end{array}$ & $\begin{array}{c}\text { select } \\
(\%)\end{array}$ & Ref. \\
\hline 1 & $\mathrm{H}_{2} \mathrm{O}, \mathrm{O}_{2}$ & $\mathrm{TiO}_{2}(\mathrm{P} 25)$ & Xe lamp, $12 \mathrm{~h}$ & benzene, $5 \mathrm{~g} ; \mathrm{H}_{2} \mathrm{O}, 50 \mathrm{~mL}$; under air & & 0.4 & & S6 \\
\hline 2 & $\mathrm{O}_{2}$ & anatase $\mathrm{TiO}_{2}$ & $\begin{array}{l}\text { high-pressure } \mathrm{Hg} \text { lamp } \\
(\lambda>350 \mathrm{~nm}), 2 \mathrm{~h}\end{array}$ & neat benzene, under air & & trace & & S7 \\
\hline 3 & $\mathrm{H}_{2} \mathrm{O}, \mathrm{O}_{2}$ & $\mathrm{Pt} / \mathrm{TiO}_{2}$ & Xe lamp, $18.5 \mathrm{~h}$ & benzene, $5 \mathrm{~g} ; \mathrm{H}_{2} \mathrm{O}, 20 \mathrm{~mL}$; under $\mathrm{O}_{2}$ & & 0.73 & & S8 \\
\hline 4 & $\mathrm{H}_{2} \mathrm{O}, \mathrm{O}_{2}$ & Fe-BEA & Xe lamp, $8 \mathrm{~h}$ & $\begin{array}{l}\text { benzene, } 10 \mathrm{mmol} \text {; } \mathrm{MeCN}, 20 \mathrm{~mL} ; \mathrm{H}_{2} \mathrm{O}, 2 \mathrm{~mL} \text {; } \mathrm{O}_{2} \text {, } \\
700 \text { Torr }\end{array}$ & & 0.035 & & S9 \\
\hline 5 & $\mathrm{H}_{2} \mathrm{O}, \mathrm{O}_{2}$ & $\mathrm{TiO}_{2}$ pillared mica & Xe lamp $(\lambda>300 \mathrm{~nm}), 2 \mathrm{~h}$ & benzene, $10 \mathrm{mmol} ; \mathrm{H}_{2} \mathrm{O}, 20 \mathrm{~mL} ; \mathrm{O}_{2}, 700$ Torr & & 0.29 & 17 & $\mathrm{~S} 10$ \\
\hline 6 & $\mathrm{H}_{2} \mathrm{O}, \mathrm{O}_{2}$ & $\mathrm{TiO}_{2} / \mathrm{ZSM}-5$ & Xe lamp $(\lambda>300 \mathrm{~nm}), 5 \mathrm{~h}$ & $\begin{array}{l}\text { aqueous solution ( } \mathrm{pH} \text { 10.9) containing } 100 \text { ppm } \\
\text { benzene, } 100 \mathrm{~mL} \text {; under } \mathrm{O}_{2}\end{array}$ & 70 & 15 & 21 & $\mathrm{~S} 11$ \\
\hline 7 & $\mathrm{H}_{2} \mathrm{O}$ & $m \mathrm{TiO}_{2}(61)$ & $\begin{array}{l}\text { high-pressure Hg lamp } \\
(\lambda>320 \mathrm{~nm}) ; 2 \mathrm{~h}\end{array}$ & $\mathrm{H}_{2} \mathrm{O}, 10 \mathrm{~mL}$; benzene, $20 \mu \mathrm{mol}$; pH 7 & 23 & 19 & 83 & This work \\
\hline 8 & $\mathrm{H}_{2} \mathrm{O}$ & $m \mathrm{TiO}_{2}(61)$ & $\begin{array}{l}\text { high-pressure } \mathrm{Hg} \text { lamp } \\
(\lambda>320 \mathrm{~nm}) ; 6 \mathrm{~h}\end{array}$ & $\mathrm{H}_{2} \mathrm{O}, 10 \mathrm{~mL}$; benzene, 20 mmol; pH 7 & 42 & 34 & 81 & This work \\
\hline 9 & $\mathrm{H}_{2} \mathrm{O}$ & $m \mathrm{TiO}_{2}(61)$ & $\begin{array}{l}\text { high-pressure Hg lamp } \\
(\lambda>320 \mathrm{~nm}) ; 6 \mathrm{~h}\end{array}$ & $\mathrm{H}_{2} \mathrm{O}, 10 \mathrm{~mL}$; benzene, $0.5 \mathrm{mmol} ; \mathrm{pH} 7$ & 10 & 8 & 80 & This work \\
\hline
\end{tabular}

Table S3. Examples of catalytic hydroxylation of benzene to phenol.

\begin{tabular}{|c|c|c|c|c|c|c|c|c|}
\hline entry & oxidant & catalyst & $\begin{array}{c}\text { reaction } \\
\text { conditions }\end{array}$ & reaction mixture composition & $\begin{array}{c}\text { conv } \\
(\%)\end{array}$ & $\begin{array}{c}\text { yield } \\
(\%)\end{array}$ & $\begin{array}{c}\text { select } \\
(\%)\end{array}$ & Ref. \\
\hline 1 & $\mathrm{~N}_{2} \mathrm{O}$ & Fe-ZSM-5 & $623 \mathrm{~K}$ & $\mathrm{~N}_{2} \mathrm{O} /$ benzene $=4$ & 27 & 26 & 98 & $\mathrm{~S} 12$ \\
\hline 2 & $\mathrm{~N}_{2} \mathrm{O}$ & Fe-ZSM-5 & $691 \mathrm{~K}$ & $\mathrm{~N}_{2} \mathrm{O} /$ benzene $=0.066$ & 4.2 & 4.0 & 97 & $\mathrm{~S} 13$ \\
\hline 3 & $\mathrm{~N}_{2} \mathrm{O}$ & ZSM-5 & $755 \mathrm{~K}$ & $\mathrm{~N}_{2} \mathrm{O} /$ benzene $=0.055$ & $4.2-4.3$ & $4.0-4.1$ & 96 & S14 \\
\hline 4 & $\mathrm{O}_{2}+\mathrm{H}_{2}$ & $\mathrm{CuSO}_{4}+\mathrm{Pd} / \mathrm{SiO}_{2}$ & room temp. & $\begin{array}{l}\text { benzene, } 1 \mathrm{~g} \text {; aqueous } \mathrm{H}_{2} \mathrm{SO}_{4}(0.05 \mathrm{M}) \text { solution, } 25 \\
\mathrm{~mL} ; \mathrm{MeCN}, 2.5 \mathrm{~mL} ; \mathrm{H}_{2} \text { is required for catalyst } \\
\text { regeneration }\end{array}$ & & 0.35 & $70-80$ & $\mathrm{~S} 15$ \\
\hline 5 & $\mathrm{O}_{2}+\mathrm{H}_{2}$ & $\mathrm{CuSO}_{4}+\mathrm{Pd} / \mathrm{SiO}_{2}$ & $323 \mathrm{~K}$ & neat benzene; $\mathrm{O}_{2} / \mathrm{H}_{2}=1$ & & 0.036 & $>90$ & $\mathrm{~S} 16$ \\
\hline
\end{tabular}


$6 \quad \mathrm{O}_{2}+\mathrm{H}_{2} \quad$ Pd/TS-1

$\mathrm{O}_{2}+\mathrm{H}_{2} \mathrm{~V} / \mathrm{M} / \mathrm{SiO}_{2}(\mathrm{M}=\mathrm{Ru}, \mathrm{Rh}, \mathrm{Pd}, \mathrm{Ir}$, $\mathrm{Pt})$

$333 \mathrm{~K}$

$298 \mathrm{~K}$;

electrochemical

$298 \mathrm{~K}$;

electrochemical

$423 \mathrm{~K}$

$453 \mathrm{~K}$

$\operatorname{Pd}(\operatorname{acac})_{2}+1,10$-phenanthoroline

monohydrate

$453 \mathrm{~K}$ $\mathrm{Pd}(\mathrm{acac})_{2}+1$,
monohydrate $)$

$\mathrm{O}_{2}+\mathrm{CO} \mathrm{Pd}(\mathrm{acac})_{2}+1,10$-phenanthoroline

$\mathrm{O}_{2} \quad \mathrm{H}_{6.3}\left[\mathrm{PMo}_{8.7} \mathrm{~V}_{3.3} \mathrm{O}_{40}\right]$

$403 \mathrm{~K}$

$\mathrm{O}_{2} \quad\left[\left\{\mathrm{Fe}\left(\mathrm{HBpz}_{3}\right)(\text { hfacac })\right\}_{2} \mathrm{O}\right]^{a}$

$298 \mathrm{~K}$

15

$\mathrm{O}_{2} \quad \mathrm{EuCl}_{3}$

$313 \mathrm{~K}$

$\mathrm{O}_{2} \quad \mathrm{Cu}-\mathrm{NaY}$

$\mathrm{H}_{2} \mathrm{O}_{2} \quad$ TS-1

$\mathrm{H}_{2} \mathrm{O}_{2}$ V-MCM-41

$303 \mathrm{~K}$

$300 \mathrm{~K}$

$343 \mathrm{~K}$

$\mathrm{H}_{2} \mathrm{O}_{2}$ 5-carboxy-2-methylpyrazine- $N$ -

$308 \mathrm{~K}$

$373 \mathrm{~K}$

$298 \mathrm{~K}$

$723 \mathrm{~K}$; flow reactor benzene, $10 \mathrm{~mL}$; aqueous $\mathrm{HCl}(0.03 \mathrm{M})$ solution, 5

$\mathrm{mL} ; \mathrm{O}_{2} / \mathrm{H}_{2}=4.67$

0.085

benzene, $20 \mathrm{~mL}$; AcOH, $25 \mathrm{~mL}$; $\mathrm{O}_{2} / \mathrm{H}_{2}=0.33$

$99.7-100$

$\mathrm{S} 18$

neat benzene, $\mathrm{O}_{2} / \mathrm{H}_{2}=2$

0.001

neat benzene, $\mathrm{O}_{2} / \mathrm{H}_{2}=2$

0.027

benzene $/ \mathrm{O}_{2} / \mathrm{H}_{2}=1 / 3.23 / 3.04$

85

benzene, $16 \mathrm{~mL}$; $\mathrm{AcOH}, 4 \mathrm{~mL} ; \mathrm{O}_{2}=\mathrm{CO}=15$ atm

benzene, $8 \mathrm{~mL}$; $\mathrm{AcOH}, 2 \mathrm{~mL} ; \mathrm{O}_{2}=\mathrm{CO}=15 \mathrm{~atm}$

benzene, $100 \mathrm{mmol} ; \mathrm{H}_{2} \mathrm{O}, 100 \mathrm{mmol}$; $\mathrm{AcOH}, 400$ mmol; LiAc, 10 mmol; $\mathrm{Pd}(\mathrm{acac})_{2}, 0.07$ mmol; $\mathrm{O}_{2}, 60$ atm

benzene, $1.66 \mathrm{mmol} ; \mathrm{CH}_{2} \mathrm{Cl}_{2}, 20 \mathrm{~mL} ; \mathrm{Zn}, 0.5 \mathrm{~g} ; \mathrm{O}_{2}$, 1 atm

AcOH, $2 \mathrm{~mL}$; benzene, $1 \mathrm{~mL} ; \mathrm{CH}_{2} \mathrm{Cl}_{2}, 2 \mathrm{~mL}$; $\mathrm{Zn}, 1 \mathrm{~g}$; $\mathrm{O}_{2}=1 \mathrm{~atm}$

benzene, $2 \mathrm{~mL}$; L-ascorbic acid; $4 \mathrm{mmol} ; \mathrm{O}_{2}=1 \mathrm{~atm}$

$\mathrm{H}_{2} \mathrm{O}_{2} /$ benzene $=10 \mathrm{~mol} / \mathrm{mol}$

benzene, $6 \mathrm{~mL}$; AcOH, $6 \mathrm{~mL} ; \mathrm{H}_{2} \mathrm{O}_{2} ; 2.5 \mathrm{~mL} / \mathrm{h}$

$\mathrm{CF}_{3} \mathrm{COOH}, 0.4 \mathrm{mmol} ; \mathrm{H}_{2} \mathrm{O}, 109 \mathrm{~mL} ; \mathrm{MeCN}, 109$

$\mathrm{mL}$; benzene, $22 \mathrm{~mL} ; \mathrm{H}_{2} \mathrm{O}_{2} 5.5 \mathrm{mmol} / \mathrm{h}$

benzene/sulfolane/30 \% $\mathrm{H}_{2} \mathrm{O}_{2}=2 / 8 / 0.2 \mathrm{v} / \mathrm{v}$

benzene, $10 \mathrm{~mL} ; 30 \% \mathrm{H}_{2} \mathrm{O}_{2}, 5 \mathrm{~mL}$

benzene, $1 \mathrm{~mL} / \mathrm{h}$; $\mathrm{NH}_{4} \mathrm{OH}(26 \mathrm{wt} \%), 1 \mathrm{~mL} / \mathrm{h}$; air, 10 $\mathrm{mL} / \mathrm{h}$

${ }^{a} \mathrm{HBpz}_{3}=$ hydrotris-1-pyrazolylborate; hfacac $=$ hexafluoroacetylacetone. 
Table S4. Lifetime of $\cdot \mathrm{OH}, \tau$, and its diffusion distance within the lifetime on $m \mathrm{TiO}_{2}$.

\begin{tabular}{lccccc}
\hline & $\begin{array}{c}A_{\text {int }} \\
\left(\mathrm{m}^{2} \mathrm{~g}^{-1}\right)^{a}\end{array}$ & $\begin{array}{c}V_{\mathrm{p}} \\
\left(\mathrm{mm}^{3} \mathrm{~g}^{-1}\right)^{b}\end{array}$ & $\begin{array}{c}{\left[\mathrm{TiO}_{2}\right]_{\mathrm{s}}} \\
\left(\mathrm{m}^{2} \mathrm{~L}^{-1}\right)\end{array}$ & $\begin{array}{c}\tau \\
(\mathrm{ns})\end{array}$ & $\begin{array}{c}x \\
(\mathrm{~nm})\end{array}$ \\
\hline$m \mathrm{TiO}_{2}(65)$ & 146 & 307 & $4.76 \times 10^{5}$ & 1.2 & 1.7 \\
$m \mathrm{TiO}_{2}(61)$ & 275 & 312 & $8.81 \times 10^{5}$ & 0.6 & 1.3 \\
$m \mathrm{TiO}_{2}(57)$ & 135 & 155 & $8.71 \times 10^{5}$ & 0.6 & 1.3 \\
$m \mathrm{TiO}_{2}(37)$ & 144 & 270 & $5.33 \times 10^{5}$ & 1.0 & 1.6 \\
$m \mathrm{TiO}_{2}(0)$ & 97 & 401 & $2.42 \times 10^{5}$ & 2.3 & 2.4 \\
\hline
\end{tabular}

${ }^{a}=A_{\mathrm{BET}}-A_{\text {ext }}($ Table 1$) .{ }^{b}$ determined by $\mathrm{N}_{2}$ adsorption-desorption measurement (Figure $\mathrm{S} 2$ ). 


\section{Figures}
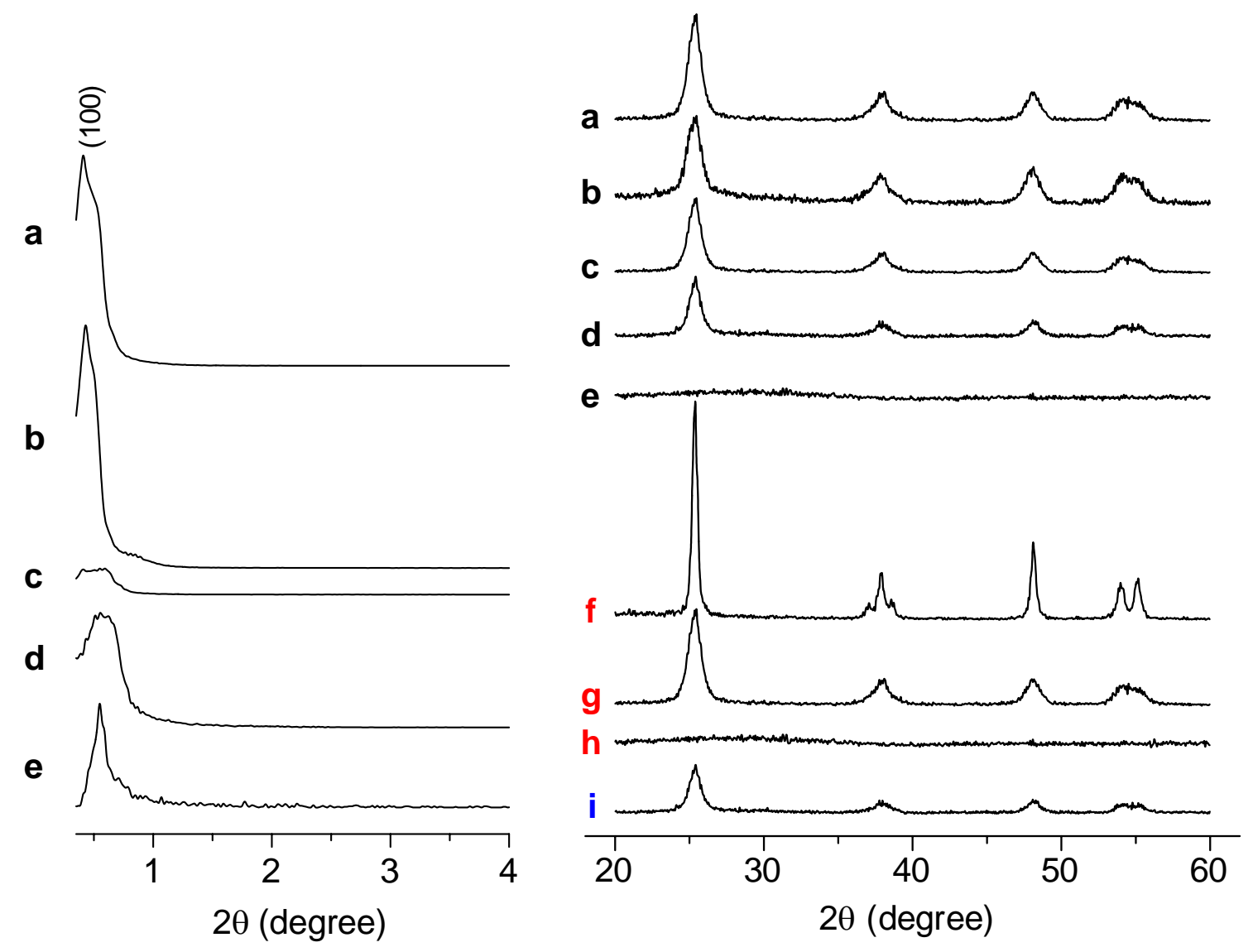

Figure S1. XRD patterns of the catalysts used. Respective catalysts are: (a) $m \mathrm{TiO}_{2}(65)$, (b) $m \mathrm{TiO}_{2}(61),(\mathbf{c}) m \mathrm{TiO}_{2}(57)$, (d) $m \mathrm{TiO}_{2}(37)$, (e) $m \mathrm{TiO}_{2}(0)$, (f) $n \mathrm{TiO}_{2}(100)$, (g) $n \mathrm{TiO}_{2}(58)$, (h) $n \mathrm{TiO}_{2}(0)$, (i) $\operatorname{agg} \mathrm{TiO}_{2}(36)$. 

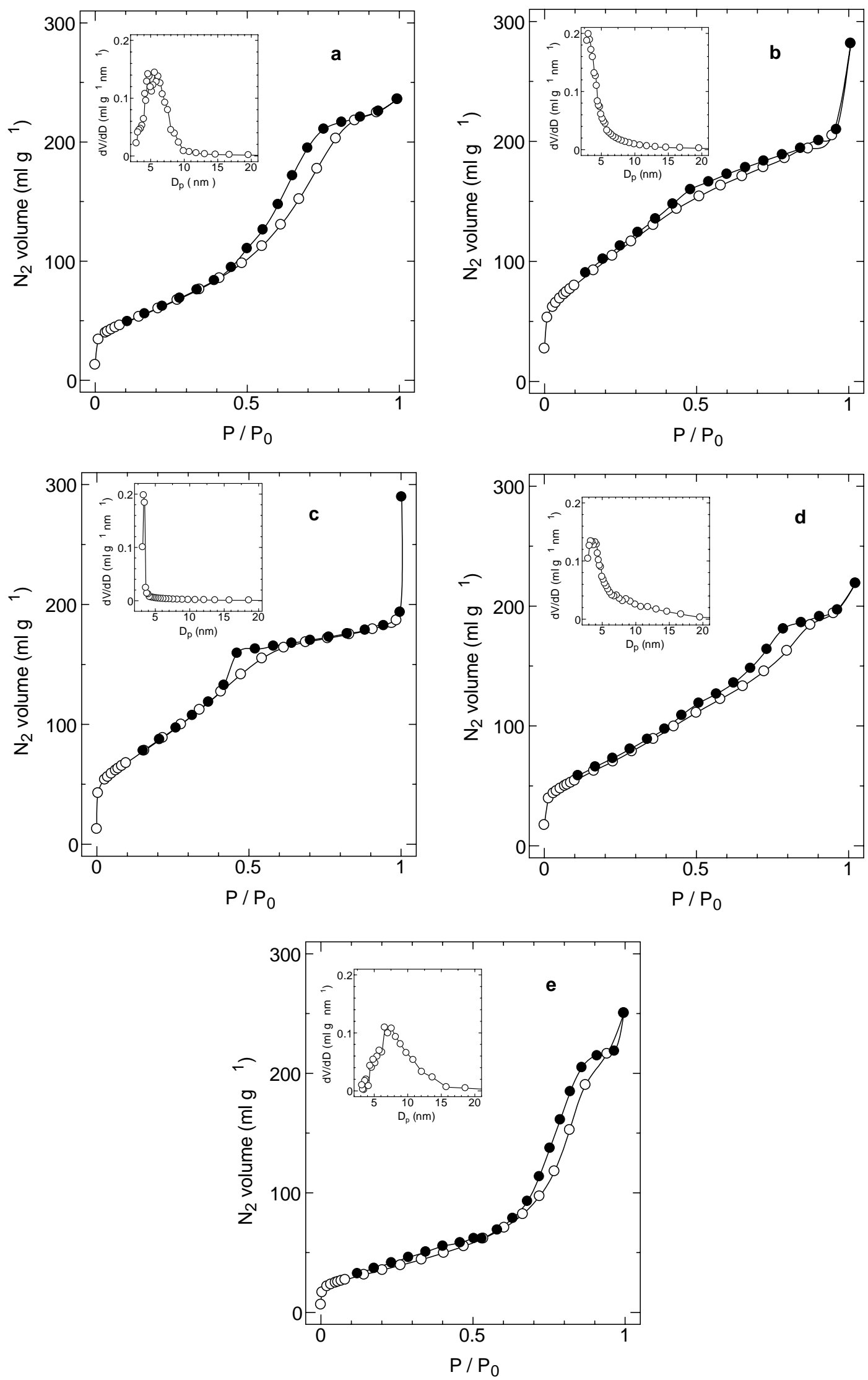

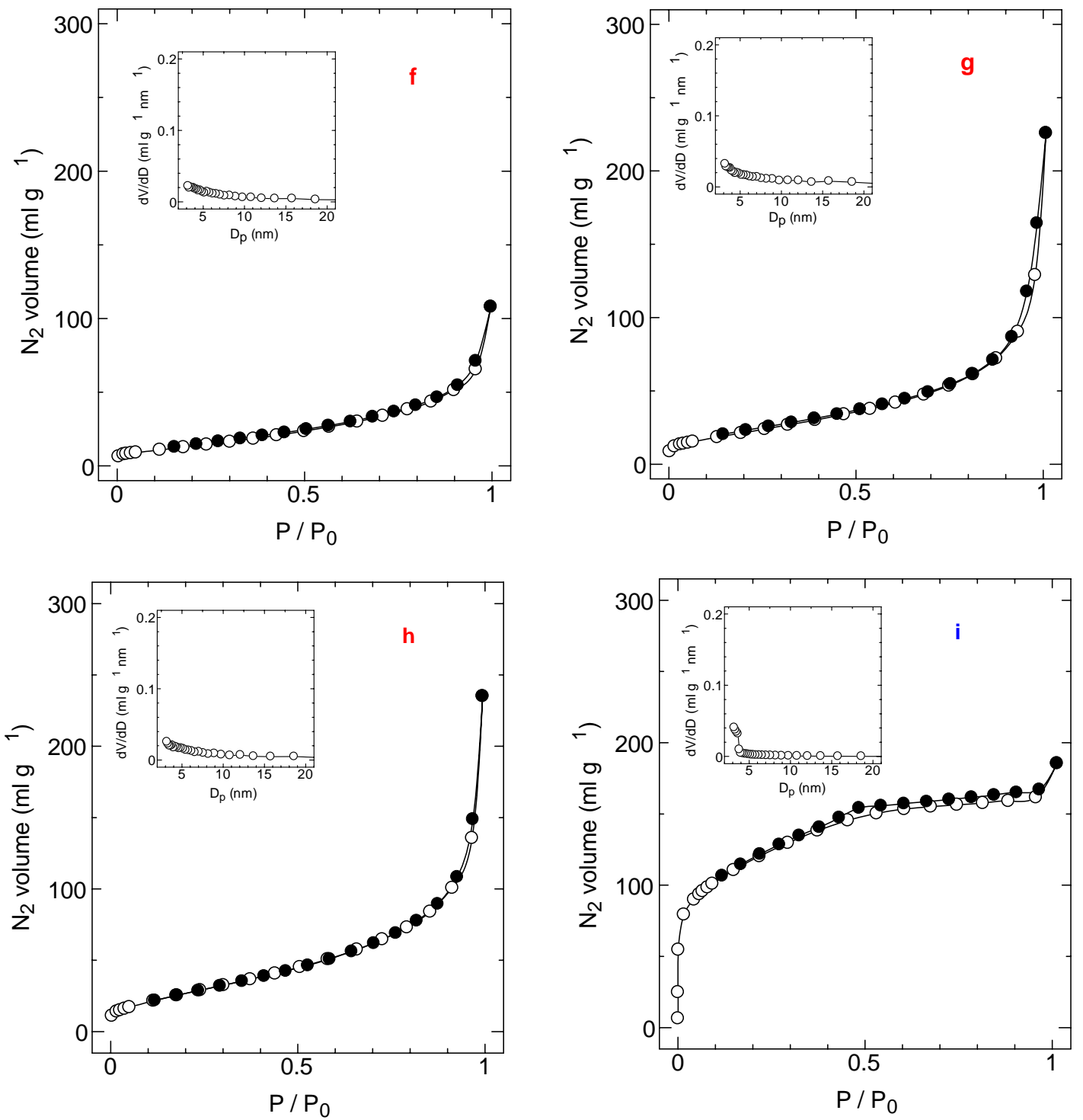

Figure S2. $\mathrm{N}_{2}$ adsorption( $(\circ)$-desorption( $\bullet$ ) isotherm and pore size distribution (inset) of the catalysts used. (a) $m \mathrm{TiO}_{2}(65),(\mathbf{b}) m \mathrm{TiO}_{2}(61)$, (c) $m \mathrm{TiO}_{2}(57)$, (d) $m \mathrm{TiO}_{2}(37)$, (e) $m \mathrm{TiO}_{2}(0),(\mathbf{f})$ $n \mathrm{TiO}_{2}(100),(\mathbf{g}) n \mathrm{TiO}_{2}(58)$, (h) $n \mathrm{TiO}_{2}(0)$, (i) $\operatorname{agg} \mathrm{TiO}_{2}(36)$.

\section{References}

(S1) Tian, B.; Yang, H.; Liu, X.; Xie, S.; Yu, C.; Fan, J.; Tu, B.; Zhao, D. Chem. Commun. 2002, 1824.

(S2) Uekawa, N.; Suzuki, M.; Ohmiya, T.; Mori, F.; Wu, Y. J.; Kakegawa, K. J. Mater. Res. 2003, 18, 797.

(S3) Lawless, D.; Serpone, N.; Meisel, D. J. Phys. Chem. 1991, 95, 5166.

(S4) Tokeshi, M.; Minagawa, T.; Kitamori, T. Anal. Chem. 2000, 72, 1711. 
(S5) Byakov, V. M.; Bugaenko, L. T.; Antonova, E. A.; Paramonov, D. V.; Trofimov, V. I. High Energy Chem. 2000, 34, 356.

(S6) Fujihira, M.; Satoh, Y.; Osa, T. Nature 1981, 293, 206.

(S7) Fujihira, M.; Satoh, Y.; Osa, T. J. Electroanal. Chem. 1981, 126, 277.

(S8) Izumi, I.; Dunn, W. W.; Wllbourn, K. O.; Fan, F.-R. F.; Bard, A. J. J. Phys. Chem. 1980, 84, 3207.

(S9) Shimizu, K.-i.; Akahane, H.; Kodama, T.; Kitayama, Y. Appl. Catal. A 2004, 269, 75.

(S10) Shimizu, K.-i.; Kaneko, T.; Fujishima, T.; Kodama, T.; Yoshida, H.; Kitayama, Y. Appl. Catal. A 2002, 225, 185.

(S11) Chen, J.; Eberlein, L.; Langford, C. H. J. Photochem. Photobiol. A 2002, 148, 183.

(S12) Panov, G. I. CATTECH 2000, 4, 18.

(S13) Bellussi, G.; Perego, C. CATTECH 2000, 4, 4.

(S14) Notté, P. P. Topics Catal. 2000, 13, 387.

(S15) Kunai, A.; Ishihata, K.; Ito, S.; Sasaki, K. Chem. Lett. 1988, 1967.

(S16) Kitano, T.; Kuroda, Y.; Itoh, A.; Jian, L.-F.; Kunai, A.; Sasaki, K. J. Chem. Soc., Perkin Trans. 2 1990, 1991.

(S17) Tatsumi, T.; Yuasa, K.; Tominaga, H. J. Chem. Soc., Chem. Commun. 1992, 1446.

(S18) Miyake, T.; Hamada, M.; Sasaki, Y.; Oguri, M. Appl. Catal. A 1995, 131, 33.

(S19) Otsuka, K.; Yamanaka, I.; Hosokawa, K. Nature, 1990, 345, 697.

(S20) Otsuka, K.; Yamanaka, I. Catal. Today 2000, 57, 71.

(S21) Niwa, S.-i.; Eswaramoorthy, M.; Nair, J.; Raj, A.; Itoh, N.; Shoji, H.; Namba, T.; Mizukami, F. Science 2002, 295, 105.

(S22) Jintoku, T.; Taniguchi, H.; Fujiwara, Y. Chem. Lett. 1987, 1865.

(S23) Jintoku, T.; Nishimura, K.; Takaki, K.; Fujiwara, Y. Chem. Lett. 1990, 1687.

(S24) Passoni, L. C.; Cruz, A. T.; Buffon, R.; Schuchardt, U. J. Mol. Catal. A 1997, 120, 117.

(S25) Moro-oka, Y.; Akita, M. Catal. Today 1998, 41, 327.

(S26) Yamanaka, I.; Nakagaki, K.; Akimoto, T.; Otsuka, K. J. Chem. Soc., Perkin Trans. 2 1996, 2511.

(S27) Ohtani, T.; Nishiyama, S.; Tsuruya, S.; Masai, M. J. Catal. 1995, 155, 158.

(S28) Thangaraj, A.; Kumar, R.; Ratnasamy, P. Appl. Catal. 1990, 57, L1.

(S29) Lee, C. W.; Lee, W. J.; Park, Y. K.; Park, S.-E. Catal. Today 2000, 61, 137.

(S30) Bianchi, D.; Bortolo, R.; Tassinari, R.; Ricci, M.; Vignola, R. Angew. Chem. Int. Ed. 2000, $39,4321$.

(S31) Balducci, L.; Bianchi, D.; Bortolo, R.; D’Aloisio, R.; Ricci, M.; Tassinari, R.; Ungarelli, R. Angew. Chem. Int. Ed., 2003, 42, 4937.

(S32) Liptáková, B.; Hronec, M.; Cvengrošová, Z. Catal. Today 2000, 61, 143. 